\title{
A Slowly Rotating Black Hole in Horava-Lifshitz Gravity and a 3+1 Dimensional Topological Black Hole: Motion of Particles and BSW Mechanism
}

\author{
Ibrar Hussain,, 4 Mubasher Jamil, ${ }^{2,+}$ and Bushra Majeed ${ }^{2, t}$ \\ ${ }^{1}$ School of Electrical Engineering and Computer Science \\ National University of Sciences and Technology \\ H-12, Islamabad, Pakistan. \\ ${ }^{2}$ School of Natural Sciences, National University of Sciences and Technology, \\ H-12, Islamabad, Pakistan.
}

\begin{abstract}
The motion of a neutral particle in the vicinity of a slowly rotating black hole in the Horava-Lifshitz theory of gravity and $3+1$ dimensional topological Lifshitz black hole is investigated. Geodesics for radial motion of the particles are also plotted. Some different cases of the orbital motion of the particle are discussed where maximum and minimum values of the effective potential are calculated. Further the Bañados, Silk and West (BSW) mechanism is studied for these black holes. It is shown that the centre-of-mass energy (CME) of two colliding uncharged particles at the horizon of these black holes remains finite. Thus the BSW effect cannot be seen in these cases.
\end{abstract}

Key words: Motion of particles; Black holes; Horava-Lifshitz gravity, 3+1 dimensional topological Lifshitz black hole; BSW mechanism.

PACS numbers: 04.70-s, 04.50.Kd

*Electronic address: ibrar.hussain@seecs.nust.edu.pk

${ }^{\dagger}$ Electronic address: mjamil@sns.nust.edu.pk

${ }^{\ddagger}$ Electronic address: bushra:majeed18@yahoo.com 


\section{INTRODUCTION}

To combine the Einstein's theory of relativity with quantum mechanics or to obtain a theory of quantum gravity (QG) is an unsolved problem in physics. In this regard several attempts have been made (for detail see [1]). In 2009 Peter Horava proposed a candidate theory for QG in 4 spacetime dimensions which is based on the explicit violation of local Lorentz invariance[2]. This is a power counting renormalizable, higher order gravity model and reduces to General Relativity (GR) at large distances. It may provide a candidate of ultraviolet completion of GR [2].

In the past few years some static and slowly rotating black hole spacetimes have been proposed in Horava-Lifshitz (HL) gravity [3 9]. In this regard Lu-Mei-Pope have studied a spherically symmetric black hole with some dynamical parameter $\lambda$ [9]. Cai et. al have obtained a topological black hole solution [5]. Another black hole solution which is asymptotically flat has been found by Kehagias and Sfetsos by taking $\lambda=1$ [3]. Besides, some slowly rotating black hole solutions in HL gravity have been investigated in literature [4, 6- 8].

Recently researchers have given a great attention to the Lifshitz spacetimes for finding the gravity duals of Lifshitz fixed points due to the AdS/CFT correspondence for condensed matter physics [10]. From the perspective of quantum field theory, there are many scale invariant theories for studying such critical points. Lifshitz field theory is a toy model which exhibits this scale invariance. Such theories exhibit the anisotropic scale invariance expressed as $t \rightarrow \lambda^{z} t$, and $x \rightarrow \lambda x$, with $z \neq 1$, where $z$ is the relative scale dimension of time and space. The Lifshitz spacetimes are given by

$$
d s^{2}=\frac{r^{2 z}}{\ell^{2 z}} d t^{2}-\frac{\ell^{2}}{r^{2}} d r^{2}-\frac{r^{2}}{\ell^{2}} d \vec{x}^{2}
$$

where $\vec{x}$ is a $D-2$ dimensional spatial vector, $D$ denotes the spacetime dimension and $\ell$ represents the length scale in the geometry. As mentioned, this spacetime is interesting due to its invariance under anisotropic scale transformation and represents the gravitational dual of strange metals [11]. When $z=1$, then (11) is the usual anti-de Sitter metric in Poincaré coordinates. The metric of the Lifshitz black hole is asymptotically similar to (1). Topological Lifshitz black hole with critical exponent $z=2$ in $3+1$ dimensions was proposed by Mann [12]. Particles motion on topological black hole in $3+1$ dimensions has been studied in [13]. 
Particle dynamics around black holes is a topic of interest for physicist for the last few decades. In this direction several papers have been appeared in the literature. Particle motion in the vicinity of a magnetized Schwarzschild black hole was studied in [14]. Particle dynamics in the Riessner-Nordstrom, Kerr and Kerr-Newman black holes was investigated by Pugliese et. al. [15-18]. An analysis of motion of particles in higher dimensional black holes has appeared in [19]. Vasudevan et. al. have investigated particle motion in Myers-Perry black hole in all dimensions [20]. The motion of particles around near-extreme Braneworld Kerr black holes has analyzed in [21]. In a regular black hole spacetime, motion of particles has discussed by Garcia et. al [22]. Particles motion in the background of a black hole in Branward geometry has investigated in [23]. Enolskii et. al. considered motion of particles in the Kehagias and Sfetsos black hole [24]. They have shown that neither massless nor massive particles with non-vanishing angular momentum can reach the singularity. The dynamics of particles around Kehagias and Sfetsos black hole immersed in an external magnetic field has studied in [25]. In [26] motion of charged particles in a rotating black hole immersed in a uniform magnetic field has considered. The influence of quintessence on the motion of particles in the vicinity of Schwarzschild black hole has discussed by Fernando [27] and Li and Zhang [28]. The tunneling radiation characteristic of charged particles from the Reissner-Nordstrm-anti de Sitter black hole has studied in [29].

Bañados, Silk and West (BSW) proposed that rotating black holes may act as particle accelerators [30]. In the vicinity of extremal Kerr black hole, they have found that infinite centre-of-mass energy (CME) can be achieved during the collision of particles. The BSW effect was then studied for different black hole spacetimes [31 50]. For a brief review one can see [46] and references therein. BSW effect for particles in the vicinity of a rotating black hole in HL gravity has studied by Abdujabbarov and Ahmedov in [25]. They have found some limitation on the CME of the accelerating particles. For another slowly rotating black hole in HL gravity the BSW mechanism has investigated by Sadeghi and Pourhassan [51]. They have obtained an infinite CME for colliding particles arbitrarily close to the horizon of the slowly rotating balck hole. Consequently in this paper we consider a slowly rotating black hole solution in HL theory of gravity given by Barausse et. al [7], and the $3+1$ dimensional topological Lifshitz black hole, to study motion of particles in the vicinity of the black holes. We also investigate the BSW process for these black hole to look at the CME of the colliding neutral particles. 
The plan of the paper is as follows. In Section 2 we formulate the equations of motion for a particle in the field of the slowly rotating black hole in the HL gravity. In subsections geodesics of the radially moving particle are plotted. We also analyze the behavior of the effective potential to look at its maximum and minimum values. Expression for CME of the colliding particles is obtained. In Section 3 we study the motion of particles in the vicinity of $3+1$ dimensional topological Lifshitz black hole. Behavior of the effective potential and CME of the colliding particles around this black hole are studied in the following subsections. We summarize our discussion in the last Section. Throughout this paper we use $G=c=1$.

\section{MOTION OF PARTICLES IN THE VICINITY OF SLOWLY ROTATING BLACK HOLE IN HORAVA-LIFSHITZ GRAVITY}

The line element for a slowly rotating black hole in HL theory of gravity is given by [7]

$$
d s^{2}=f(r) d t^{2}-\frac{B(r)^{2}}{f(r)} d r^{2}-r^{2}\left(d \theta^{2}+\sin ^{2} \theta d \phi^{2}\right)+\epsilon r^{2} \sin ^{2} \theta \Omega(r, \theta) d t d \phi+O\left(\epsilon^{2}\right),
$$

where $f(r)$ and $B(r)$ are given as a series expansion for the spherically symmetric static asymptotically flat solution in terms of the inverse radial coordinates $x=\frac{\alpha}{r}$ (for detail one can see [8]). For simplicity, we consider only those terms which are linear in $x$ and neglect the higher order terms. Therefore

$$
f(r)=1+\frac{\alpha}{r}, \quad B(r)=1
$$

where $\alpha$ is an arbitrary constant with dimensions of length. In $(2) \epsilon$ is known as the bookkeeping parameter of the expansion in the rotation. The motion of a neutral particle in the vicinity of a black hole is determined by the following Lagrangian

$$
\mathcal{L}=\frac{1}{2} g_{\mu \nu} \dot{x^{\mu}} \dot{x^{\nu}}
$$

where $g_{\mu \nu}$ is the metric tensor and over-dot denotes the derivative with respect to the geodetic parameter. Here $\mu, \nu=0,1,2,3$. Using (2) in (41), we get

$$
\mathcal{L}=\frac{1}{2}\left[f(r) \dot{t}^{2}-\frac{B(r)^{2}}{f(r)} \dot{r}^{2}-r^{2} \dot{\theta}^{2}-r^{2} \sin ^{2} \dot{\phi}^{2}+\epsilon r^{2} \sin ^{2} \theta \Omega(r, \theta) \dot{t} \dot{\phi}+O\left(\epsilon^{2}\right)\right] .
$$

The time coordinate basis $\partial / \partial t$ and azimuthal angular coordinate basis $\partial / \partial \phi$ are Killing vectors of the metric (2) we considered here, therefore conserved quantities along the geodesics 
of the particles are the energy $E$, of the particle and the angular momentum $L$, of the particle respectively. The Euler -Lagrange equations give the following relations upon integration

$$
\begin{aligned}
& \tilde{\mathcal{E}} \equiv-\frac{E}{m}=\frac{\partial \mathcal{L}}{\partial \dot{t}}=f(r) \dot{t}+\frac{\epsilon}{2}\left(r^{2} \sin ^{2} \theta\right) \Omega \dot{\phi}, \\
& \tilde{\mathcal{L}} \equiv-\frac{L}{m}=\frac{\partial \mathcal{L}}{\partial \dot{\phi}}=-r^{2} \sin ^{2} \theta \dot{\phi}+\frac{\epsilon}{2}\left(r^{2} \sin ^{2} \theta\right) \Omega \dot{t},
\end{aligned}
$$

here $m$ is mass of particle and terms of order $O(\epsilon)$ are retained while higher order terms are ignored in whole calculations. With-out loss of generality, the motion of the particles can be confined to the equatorial plane, $\theta=\pi / 2$. For $\theta=\pi / 2$ and eliminating $\dot{t}$ from (6) and (7), we get

$$
\dot{\phi}=\frac{L}{m r^{2}}-\epsilon\left(\frac{E \Omega}{2 m f}\right) .
$$

Further substituting (8) in (6), we get

$$
\dot{t}=\frac{-1}{m f}\left(E+\frac{\epsilon}{2} \Omega L\right),
$$

To find $\dot{r}$ we use the normalization condition:

$$
u^{\mu} u_{\mu}=1,
$$

here $u^{\mu}$ denotes the velocity components of the moving particles. For the metric under consideration, (10) yields

$$
f \dot{t}^{2}-\frac{\dot{r}^{2}}{f}-r^{2} \dot{\phi}^{2}+2\left(\epsilon r^{2} \Omega\right) \dot{t} \dot{\phi}=1 .
$$

Making use of (8) and (9) in (11), we get

$$
\dot{r}^{2}=f\left(\frac{E^{2}}{m^{2} f}-\frac{L^{2}}{m^{2} r^{2}}-1\right),
$$

or

$$
\frac{d r}{d \tau}=\dot{r}= \pm \sqrt{f\left(\frac{E^{2}}{m^{2} f}-\frac{L^{2}}{m^{2} r^{2}}-1\right)},
$$

here \pm sign denotes the radial velocity of the outgoing and ingoing particles respectively.

\section{A. Geodesics of a Radially Moving Particle}

If the particle is moving radially towards black hole we can get the geodesics of such particle. Using (9) and (13) together for zero angular momentum, $L=0$, we obtain

$$
\frac{d r}{d t} \equiv \frac{\dot{r}}{\dot{t}}=\mp \frac{\sqrt{\mathcal{E}^{2}-f(r)}}{\mathcal{E} f^{-1}(r)},
$$




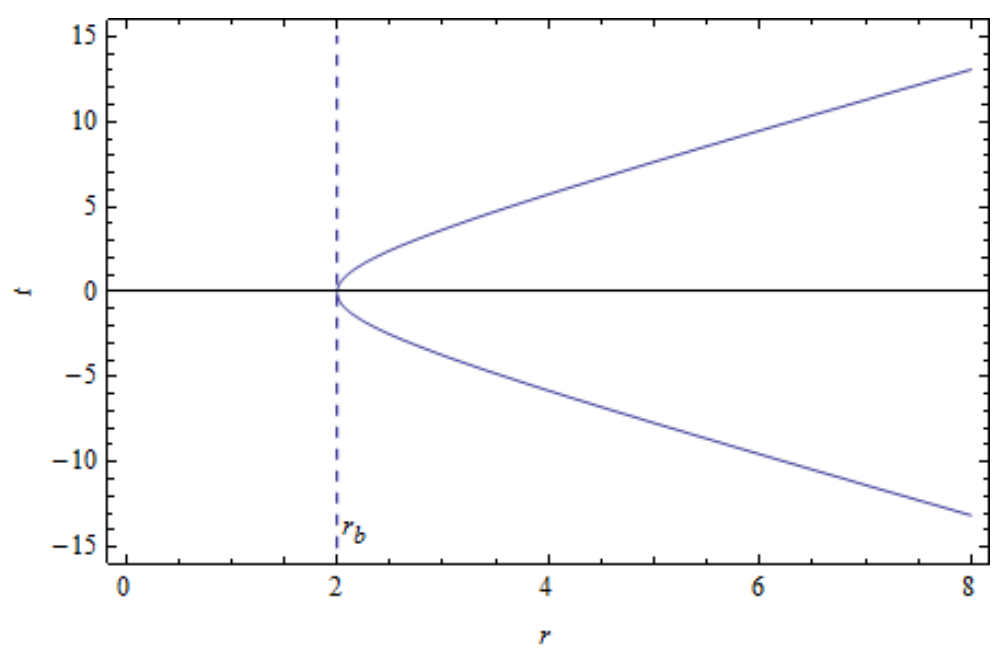

FIG. 1: Geodesics for a radially ingoing particle coming from infinity with some initial velocity, reaching $r=r_{b}$ (dashed line), and going back to infinity. We chose $\alpha=1, \mathcal{E}^{2}=1.5$.

where $\mathcal{E} \equiv-E / m$. Changing position of a radially moving particle with the passage of time could be obtained from (14), where positive root gives the path of the particle going away from black hole, and negative root gives the path of an ingoing particle. Note that (14) is defined only if $\mathcal{E}^{2}>f(r)$, or $r>\alpha /\left(\mathcal{E}^{2}-1\right)$. If we choose $\mathcal{E}^{2}=1.5$, then there is a boundary, $r_{b}$,

$$
r_{b} \equiv r=\frac{\alpha}{0.5}
$$

beyond which the particle can not go. Geodesics defined in (14) could be understood better by plotting in $(r, t)$ coordinates, Fig(1).

\section{B. Circular Orbits and Behavior of Effective Potential}

Here we analyze the effective potential to look at the stable and unstable circular orbits of the moving particles. It is known that the possible range for the motion of the particle is given by $E^{2} \geq V_{\text {eff }}^{2}$ (see for example [28]). Further the orbits of massive particles can be circular if the $V_{\text {eff }}$ attains it maximum and minimum values. It means that for the minimum value of $V_{\text {eff }}$ the circular orbits are stable and for the maximum value of $V_{\text {eff }}$ the circular orbits are unstable. For inflection point $V^{\prime \prime}=0$, yields the marginally stable orbit,

We can write (12) as below

$$
\dot{r}^{2}=\frac{1}{m^{2} B^{2}}\left(E^{2}-V_{\text {eff }}\right),
$$


where

$$
V_{\text {eff }}=f\left(\frac{L^{2}}{r^{2}}+m^{2}\right)
$$

is the effective potential and

$$
V_{\mathrm{eff}}^{\prime} \equiv \frac{d V_{\mathrm{eff}}}{d r}=\frac{-m^{2} r^{2} \alpha-L^{2}(2 r+3 \alpha)}{r^{4}} .
$$

Solving $V_{\text {eff }}^{\prime}=0$, for $r$ we get

$$
r_{ \pm}=\frac{-L^{2} \pm \sqrt{L^{4}-3 L^{2} m^{2} \alpha^{2}}}{m^{2} \alpha} .
$$

For real values of $r, L^{2} \geq 3 m^{2} \alpha^{2}$ is required. Also

$$
V_{\mathrm{eff}}^{\prime \prime} \equiv \frac{d^{2} V_{\mathrm{eff}}}{d r^{2}}=\frac{2\left(m^{2} r^{2} \alpha+3 L^{2}(r+2 \alpha)\right)}{r^{5}} .
$$

Now we check the behavior of $V_{\text {eff }}$ at the above obtained radii given by (19). Note that at $L^{2}=3 m^{2} \alpha^{2}$, particle has a circular orbit, of radius

$$
\left|r_{o}\right|=3 \alpha,
$$

and

$$
\left.V_{\text {eff }}^{\prime \prime}\right|_{r=r_{o}}=0 .
$$

Behavior of $V_{\text {eff }}$ with changing value of radius, $r$, is demonstrated in Fig. (2).

If $L^{2}>3 m^{2} \alpha^{2}$ then

$$
\left.V_{\mathrm{eff}}^{\prime \prime}\right|_{r=r_{+}}=\frac{2 L^{2} m^{8} \alpha^{4}\left(-L^{2}+3 m^{2} \alpha^{2}+\sqrt{L^{4}-3 L^{2} m^{2} \alpha^{2}}\right)}{\left(-L^{2}+\sqrt{L^{4}-3 L^{2} m^{2} \alpha^{2}}\right)^{5}},
$$

and

$$
\left.V_{\text {eff }}^{\prime \prime}\right|_{r=r_{-}}=\frac{2 L^{2} m^{8} \alpha^{4}\left(L^{2}-3 m^{2} \alpha^{2}+\sqrt{L^{4}-3 L^{2} m^{2} \alpha^{2}}\right)}{\left(L^{2}+\sqrt{L^{4}-3 L^{2} m^{2} \alpha^{2}}\right)^{5}} .
$$

Let $L^{2}-3 \alpha^{2} m^{2}=\beta^{2}$ the above expressions (21) and (22) reduce to:

$$
\left.V_{\mathrm{eff}}^{\prime \prime}\right|_{r=r_{+}}=\frac{2 L^{2} m^{8} \alpha^{4}\left(-\beta^{2}+\beta L\right)}{\left(-L^{2}+\beta L\right)^{5}},
$$

and

$$
\left.V_{\mathrm{eff}}^{\prime \prime}\right|_{r=r_{-}}=\frac{2 L^{2} m^{8} \alpha^{4}\left(\beta^{2}+\beta L\right)}{\left(L^{2}+\beta L\right)^{5}},
$$




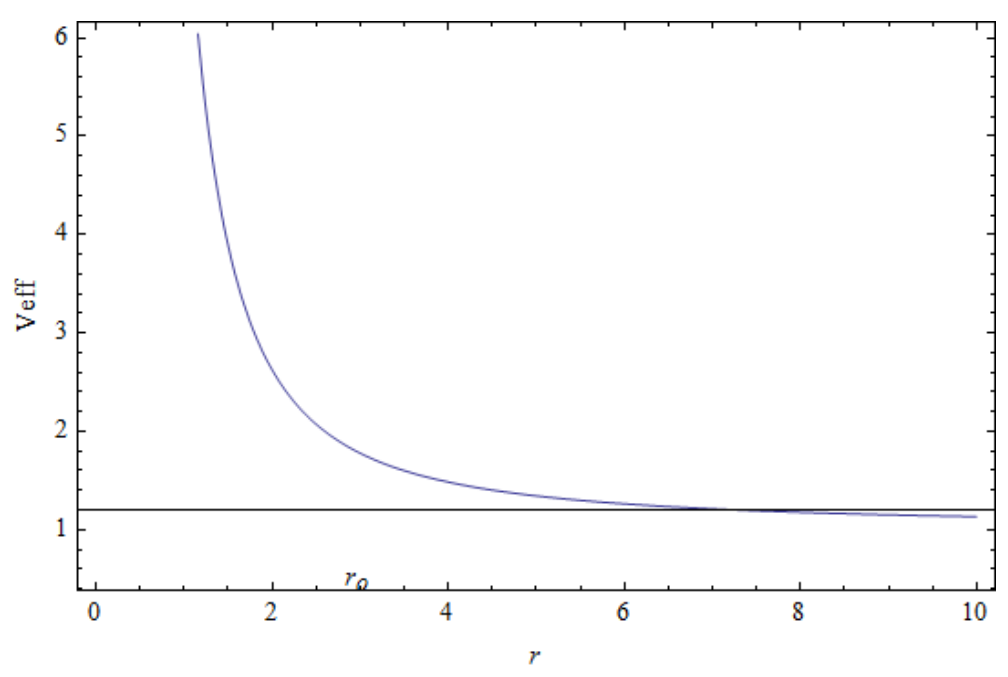

FIG. 2: Evolution of effective potential versus radius $r$, parameters values are set as, $L^{2}=3, \alpha=1$, $m=1$. At $r=r_{o}=3$ there is a circular orbit of the particle.

We see that quantity in the R.H.S. of (23) is greater than zero if

$$
\beta L>\beta^{2}, \quad \beta L>L^{2}
$$

or if

$$
\beta^{2}>\beta L, \quad L^{2}>L \beta
$$

but both conditions lead to the contradiction:

$$
\beta>L>\beta
$$

Hence $V_{\text {eff }}$ does not attain minimum value at $r=r_{+}$. The $V_{\text {eff }}$ has maximum at $r_{+}$if $\beta<L$ (which gives $3 \alpha^{2} m^{2}>0$, a physically true constraint) therefore the circular orbits are unstable.

Now there are two possible cases for $\left.V_{\mathrm{eff}}^{\prime \prime}\right|_{r=r_{-}}>0$.

Case 1:

When both $\beta^{2}+\beta L$ and $L^{2}+\beta L$ are positive quantities which give $\beta>-L$ or $3 m^{2} \alpha^{2}<0$ which is a contradiction so we exclude this case.

Case 2:

When both $\beta^{2}+\beta L<0$ and $L^{2}+\beta L<0$. This implies $\beta<-L$ or $3 m^{2} \alpha^{2}>0$, which is physically true. So $\left.V_{\text {eff }}\right|_{r=r_{-}}>0$, if $\beta<-L$. Hence $V_{\text {eff }}$ is minimum at $r=r_{-}$if $\beta<-L$ and the circular orbits are stable. 


\section{Center of Mass Energy}

Energy in the center of mass frame is defined as [30]

$$
E_{c m}=m \sqrt{2} \sqrt{1-g_{\mu \nu} u_{1}^{\mu} u_{2}^{\nu}}
$$

where

$$
u_{i}^{\mu} \equiv \frac{d x^{\mu}}{d \tau}, \quad i=1,2
$$

is the 4 -velocity of each of the particle. Using (8), (9), and (13), ((13) with negative sign as the particle coming towards the black hole), in (26), we get the CME, for the neutral particle falling freely from rest at infinity as

$$
\begin{aligned}
E_{c m}= & A\left[1-\frac{E^{2}}{m^{2} f}+\frac{1}{m^{2} f}\left\{E^{4}+\frac{f}{r^{2}}\left(L_{1}^{2}+L_{2}^{2}\right)\left(m^{2} f-E^{2}\right)\right.\right. \\
& \left.\left.-f m^{2}\left(2 E^{2}-f m^{2}\right)+\frac{L_{1}^{2} L_{2}^{2} f^{2}}{r^{4}}\right\}^{1 / 2}+\frac{L_{1} L_{2}}{m^{2} r^{2}}\right]^{1 / 2},
\end{aligned}
$$

here $A=m \sqrt{2}$. We observe that near the horizon (i.e. at $f(r)=0), E_{c m}$ in (28) becomes undefined. However expanding the numerator in (28), about the horizon yields

$$
E_{c m}=2 m \sqrt{1+\frac{1}{4 m^{2} \alpha^{2}}\left(L_{1}+L_{2}\right)^{2}} .
$$

The CME in (29) could be infinite, if the angular momentum of one of the particles has infinite value, for which the particle could not reach the horizon of the black hole. Thus the CME in (29) can not be unlimited.

\section{MOTION OF THE PARTICLES IN THE VICINITY OF $3+1$ DIMENSIONAL TOPOLOGICAL BLACK HOLE}

The metric of four dimensional Lifshitz black hole is given by [12]

$$
d s^{2}=\frac{r^{2} f(r)}{\ell^{2}} d t^{2}-\frac{d r^{2}}{f(r)}-r^{2}\left(d \theta^{2}+\sinh ^{2} \theta d \phi^{2}\right),
$$

where

$$
f(r)=\frac{r^{2}}{\ell^{2}}-\frac{1}{2}
$$

$f(r)=0$, gives the event horizon at $r_{h}=\ell / \sqrt{2}$, where $\ell$ denotes the length scale in the geometry. To find out the CME of the colliding particles in the vicinity of Lifshitz black 
hole, we first find out the geodesics structure of the particles. Using the standard Lagrangian procedure we find the conserved quantities of the moving particles of mass $m\left(m=m_{1}=\right.$ $\left.m_{2}\right)$. For each particle the velocity components are given by

$$
\dot{t}=-\sqrt{\frac{E}{m}} \frac{\ell^{2}}{f r^{2}},
$$

and

$$
\dot{\phi}=\frac{L}{m r^{2}} \text {. }
$$

The normalization condition given in (10), used for the metric defined in (30) gives

$$
\dot{r}^{2}=\frac{\ell^{2}}{m r^{2}}\left(E-V_{\mathrm{eff}}\right)
$$

or

$$
\dot{r}= \pm \frac{\ell}{r \sqrt{m}} \sqrt{E-V_{\text {eff }}},
$$

the \pm signs stand for radial velocities of outgoing and ingoing particles respectively, and $V_{\text {eff }}$ is defined as

$$
V_{\text {eff }}=\frac{f r^{2}}{\ell^{2}}\left[m+\frac{L^{2}}{m r^{2}}\right] .
$$

This expression for $V_{\text {eff }}$ is already derived in literature [13], we rederive it using dimensionally correct equations (32) and (33). Geodesics of a radially moving particle for this black hole are also already plotted in [13].

\section{A. Behavior of Effective Potential}

Differentiating (36) with respect to $r$, we have

$$
V_{\mathrm{eff}}^{\prime}=\frac{r\left(2 L^{2}-m^{2}\left(\ell^{2}-4 r^{2}\right)\right)}{\ell^{4} m} .
$$

Solving $V_{\text {eff }}^{\prime}=0$, for $r$ we get

$$
r=0, \quad r_{ \pm}= \pm \frac{\sqrt{-2 L^{2}+\ell^{2} m^{2}}}{2 m}
$$

Furthermore,

$$
\frac{d^{2} V_{\mathrm{eff}}}{d r^{2}} \equiv V_{\mathrm{eff}}^{\prime \prime}=\frac{2 L^{2}-m^{2}\left(\ell^{2}-12 r^{2}\right)}{\ell^{4} m}
$$

We evaluate (39) at above obtained radii given in (38), to check the nature of $V_{\text {eff. At }} r=r_{ \pm}$, (39) becomes

$$
\frac{d^{2} V_{\mathrm{eff}}}{d r^{2}} \equiv V_{\mathrm{eff}}^{\prime \prime}=\frac{4 L^{2}}{m \ell^{4}}+\frac{2 m}{\ell^{2}}
$$


Note that for stable orbit $V_{\text {eff }}^{\prime \prime}>0$ then

$$
\frac{m^{2} \ell^{2}}{2}>L^{2}
$$

It shows that $V_{\text {eff }}$ is minimum at $r=r_{ \pm}$, for $L^{2}<\ell^{2} / 2$ and the two stable circular orbits exist. Choosing $\ell=10$ as in [13], we get $-\sqrt{50}<L<\sqrt{50}$. If the particles approach the black hole from opposite directions then their angular momentums would have opposite sign.

\section{B. Center of Mass Energy}

For two neutral particles falling freely from rest at infinity the CME in the vicinity of $3+1$ dimensional topological Lifshitz black hole is calculated by using (32), (33) and (35) (in (35) we choose negative sign only, and its reason was explained before in section 2) in equation of $\mathrm{CME}$, we get

$$
E_{c m}=m \sqrt{2}\left[\left(L_{1}^{2}+L_{2}^{2}\right)\left(\frac{-1}{2 r^{2} m^{2}}+\frac{f}{2 m E \ell^{2}}\right)+\frac{L_{1} L_{2}}{m^{2} r^{2}}\left(1+\frac{f L_{1} L_{2}}{2 m E \ell^{2}}\right)+\frac{f r^{2} m}{2 E \ell^{2}}\right]^{1 / 2} .
$$

Expanding the numerator in (40) at the horizon of the black we obtain

$$
E_{c m}=\frac{\sqrt{2}}{\ell}\left(L_{1}-L_{2}\right)
$$

Note that for the CME to be positive $L_{1}>L_{2}$. The CME in (41) goes to infinity if $L_{1}$ attain infinite value, for which the particle could not reach the black hole horizon, hence in this case CME will remains finite.

\section{CONCLUSIONS}

In the background of a slowly rotating black hole in the HL theory of gravity and $3+1$ dimensional topological Lifshitz black hole we have analyzed the motion of massive (neutral) particles, falling freely from infinity with zero velocity there. Analytical expressions for effective potentials and the CME of neutral particles have obtained by using the standard Lagrangian methods. Geodesics of the particle coming from infinity, with some initial velocity, approaching black hole radially, has also plotted in $(r, t)$ coordinates. It has observed that in the case of slowly rotating black hole in HL gravity the particle coming from infinity 
approaches the black hole and a smooth curve has obtained which ends at the boundary $r=r_{b}=2$. Then we have combined that curve with the curve obtained for the particle moving away from the black hole, Fig. (11) has obtained.

We have examined the behavior of effective potentials in both cases of black holes. Calculations have shown that in the case of slowly rotating black hole in the HL gravity, $V_{\text {eff }}$ in (21), does not attain minimum value at $r=r_{+}$given by (19) and is maximum if $\beta<L$ is satisfied. It shows that the unstable circular orbits of the particle exist there. While $V_{\text {eff }}$ in (22) is minimum at $r=r_{-}$if $\beta<-L$. In this case there exists stable circular orbits of the moving particles. For the $3+1$ dimensional topological Lifshitz black hole $V_{\text {eff }}$ in (36) is minimum at both $r=r_{ \pm}$given by (38), for $-\sqrt{50}<L<\sqrt{50}$ and the circular orbits are stable. We plot the effective potential of test particle moving around slowly rotating black hole in HL gravity, as a function of $r$, by choosing the appropriate values of parameters.

We also found the expressions for the collision energy in the CM frame of neutral particles in the vicinity of these black holes. It is observed that the CME of the neutral particles falling from rest at infinity will remain finite in the CM frame at the horizon of these black holes. Thus the BSW effect can not be seen for the two cases of the black holes discussed here.

[1] C. Rovelli, Quantum Gravity, (Cambridge University Press, 2004).

[2] P. Horava, Phys. Rev. D 79, 084008 (2009).

[3] A. Kehagias and K. Sfetsos, Phys. Lett. B 678, 123 (2009).

[4] H. Lee, Y. Kim and Y.S. Myung, Eur. Phys. J. C 70, 361 (2010).

[5] R. G. Cai, L. M. Cao and N. Ohta, Phys. Lett. B 679, 504 (2009).

[6] E. Barausse and T. P. Sotiriou, Phys. Rev. Lett. 109, 181101 (2013).

[7] E. Barausse and T. P. Sotiriou, Phys. Rev. D 87, 087504 (2013).

[8] E. Barausse, T. Jacobson and T. P. Sotiriou, Phys. Rev. D 84, 124043 (2011).

[9] H. Lu, J. Mei and C. N. Pope, Phys. Rev. Lett. 103, 091301 (2009).

[10] S. Kachru, X. Liu and M. Mulligan, Phys. Rev. D 78, 106005 (2008).

[11] S. A. Hartnoll, J. Polchinski, E. Silverstein and D. Tong, J. High Energy Phys. 1004, 120 (2010). 
[12] R. B. Mann and J. High Energy Phys. 06, 075 (2009).

[13] M. Olivares, G. Rojas and Y. Vasquez and J. R. Villanueva, Astrophys. Space. Sci. 347, 83 (2013).

[14] A. M. Al Zahrani, V. P. Frolov and A. A. Shoom, Phys. Rev. D 87, 084043 (2013).

[15] D. Pugliese, H. Quevedo, and R. Ruffini, Phys. Rev. D 83, 024021 (2011).

[16] D. Pugliese, H. Quevedo, and R. Ruffini, Phys. Rev. D 83, 104052 (2011).

[17] D. Pugliese, H. Quevedo, and R. Ruffini, Phys. Rev. D 84, 044030 (2011).

[18] D. Pugliese, H. Quevedo, and R. Ruffini, Phys. Rev. D 88, 024042 (2013).

[19] V. Frolov and D. Stojkovic Phys. Rev. D 68, 064011 (2013).

[20] M. Vasudevan, K. A. Stevens and D. N. Page, Class. Quant. Grav. 22, 1469 (2005).

[21] Z. Stuchlik, M. Blaschke and P. Slany, Class. Quant. Grav. 28, 175002 (2011).

[22] A. Garcia, E. Hackmann, J. Kunz, C. Lmmerzahl and A. Macias, arXiv:1306.2549 [gr-qc].

[23] A. Abdujabbarov and B. Ahmedov, Phys. Rev. D 81, 044022 (2010).

[24] V. Enolskii, B. Hartmann, V. Kagramanova, J. Kunz, C. Lammerzahl, and P. Sirimachan, Phys. Rev. D 84, 084011 (2011).

[25] A. Abdujabbarov, B. Ahmedov and A. Hakimov, Phys. Rev. D 83, 044053 (2011).

[26] A. Abdujabbarov, B. Ahmedov and N. B. Jurayeva, Phys. Rev. D 87, 064042 (2013).

[27] S. Fernando, Gen. Relativ. Gravit. 44, 1857 (2012).

[28] E. Li and Y. Zhang, Astrophys. Space Sci. 350, 361 (2014).

[29] D. Y. Chen, Q. Q. Jiang and S. Z. Yang, Int. J. Theo. Phys. 46, 3275 (2007).

[30] M. Banados, J. Silk, S. M West, Phys. Rev. Lett. 103, 111102 (2009).

[31] T. Jacobson, T. P.Sotiriou, Phys. Rev. Lett. 104, 021101 (2010).

[32] O. B. Zaslavskii, JETP Lett. 92, 571 (2010).

[33] A. A. Grib and Y. V. Pavlov, JETP Lett. 92, 125 (2010).

[34] S. W. Wei, Y. X. Liu, H. Guo and C. E Fu, Phys. Rev. D 82, 103005 (2010).

[35] K. Lake, Phys. rev. Lett. 104211102 (2010).

[36] O. B. Zaslavskii, Phys. Rev. D 81, 044020 (2010).

[37] S. W. Wei, Y.X. Liu, H. Guo, Chun-E Fu, Phys. Rev. D 82, 103005 (2010).

[38] S.W. Wei, Y.X. Liu, H. Guo, Chun-E Fu, JHEP 1012066 (2010).

[39] Y. Zhu, S. Wu, Y. Jiang, G. Yang, arXiv:1108.1843.

[40] A. Williams, Phys. Rev. D 83, 123004 (2011). 
[41] M. Patil, A. S. Joshi, K. Nakao, M. Kimura, arXiv: 1108.0288.

[42] S. Gao and C. Zhong, Phys. Rev. D 84, 044006 (2011).

[43] P. J. Mao, R. Li, L. Y. Jia and J. R. Ren, arXiv:1008.2660v3.

[44] C. Zhong and S. Gao, JETP Lett. 94, 631 (2011).

[45] E. Berti, V.Cardoso, L.Gualtieri, F. Pretorius, and U. Sperhake, Phys. Rev. Lett. 103, 239001 (2009).

[46] I. Hussain, Mod. Phys. Lett. A, 27, 1250017 (2012)

[47] I. Hussain, Mod. Phys. Lett. A, 27, 1250068 (2012).

[48] I. Hussain, J .Phys. Conf. Scr. 354, 012007 (2012).

[49] M. Sharif and N. Haider, Astrophys. Space Sci. 346, 111 (2013).

[50] M. Sharif and N. Haider, J. Exp. Theo. Phys. 117, 78 (2013).

[51] J. Sadeghi and B. Pourhassan, Eur. Phys. J. C 72, 1984 (2012). 